\title{
Association of N on Alcoholic Fatty Liver with type 2 Diabetes Mellitus
}

\author{
Shobha Luxmi, Rukhsana Abdul Sattar and Jamal Ara
}

\begin{abstract}
BACKGROUND: Non alcoholic fatty liver disease (NAFLD) is a common chronic condition of which Diabetic fatty liver accounts for a large proportion, with 50 to $\mathbf{7 5 \%}$ of the subjects demonstrating fat in the liver on Ultrasound. As a result of epidemic increase in obesity, hyperlipidemia and our diabetes mellitus patients, the prevalence of NAFLD in the general population is increasing. The aim of this study is to determine the frequency of NAFLD in type 2 diabetic patients in our setup at Jinnah postgraduate Medical Center,Karachi Pakistan.

METHODS: A total of $\mathbf{1 2 0}$ type $\mathbf{2}$ diabetic patients were included in the study. Patients with known chronic liver disease and history of alcohol intake were excluded. These patients were evaluated by abdominal ultrasonography to determine the presence of fatty liver. They were divided into fatty liver group and non fatty liver group; and were further evaluated by measurement of body mass index, HbA1c, liver function tests and lipid profile. The data obtained was analyzed using SPSS version 10.0

RESULTS: Out of 120 type 2 diabetic patients, 73 (60.8 \%) had fatty liver on ultrasonography. An increase in the BMI and levels of HBA1c, ALT, AST, alkaline phosphatase and GGT, total cholesterol, triglycerides, LDL and a decrease in HDL was observed in the fatty liver group as compared to non fatty liver group.

CONCLUSION: The prevalence of NAFL is higher in type-2 diabetic patients. Obesity, dysglycemia, dyslipidemia and elevated liver enzymes are seen more frequently in fatty liver than nonfatty liver type-2 diabetic patients
\end{abstract}

KEYWORDS: Type-2 diabetes mellitus, NAFLD, BMI, Triglycerides, LFT.

\section{INTRODUCTION}

Fatty liver or hepatic steatosis is characterized by diffuse accumulation of fat in hepatocytes. ${ }^{1}$ Fatty liver occurring in individuals without a history of significant alcohol intake is termed as non-alcoholic fatty liver disease (NAFLD). ${ }^{2,3}$ The natural history of NAFLD ranges from pure steatosis to steatohepatitis ${ }^{4}$ to cirrhosis $[5,6]$ and in some patients to hepatocellular carcinoma. ${ }^{7}$ NAFLD is strongly associated with obesity, type-2 diabetes mellitus and hyperlipidema.[8,9] Numerous studies show that it is hepatic component of metabolic syndrome[2,8] whose central features are peripheral insulin resistance, obesity, hyperinsulinemia, hypertriglyceridemia and hypertension. ${ }^{8,10}$ It has been reported that fatty liver influences the severity of hepatic insulin resistance in type-2 diabetes mellitus. ${ }^{11}$ The hepatic fat content predicts the amount of daily insulin needed to maintain adequate glycemic control. ${ }^{12}$ NAFLD, is a type of chronic liver disorder which is gaining significant importance worldwide.,13 It is now recognized as the most common liver disorder in the United States ${ }^{14}$ with an estimated prevalence of $30 \%$ in the general population. ${ }^{2}$ The prevalence is reported higher among patients with diabetes mellitus and obesity ranging from $35 \%$ to $75 \%$ in various studies. ${ }^{15,16,17,18}$ The prevalence of NAFLD is also on rise in Pakistan and other Asian countries which may be attributed to the improvement of life style, the change of dietetic structure and the application of new diagnostic technique. ${ }^{17,19,20}$ Most of the patients with NAFLD have minor symptoms at presentation. They may have fatigue, malaise or sensation of fullness or discomfort in right upper quadrant of abdomen. Hepatomegaly is the only finding in the majority. ${ }^{20,21}$ The definitive diagnosis of NAFLD is based on the histologic examination of liver biopsy samples. However, it is an invasive and costly procedure and is associated with many complications. Radiologic imaging of the liver with sonography, computed tomography or magnetic resonance imaging has an adequate threshold for the detection of fatty liver. Ultrasound is usually the most widely available and cheap test performed and has $80 \%$ sensitivity and $99 \%$ specificity. ${ }^{17,22}$ The sonographic features of NAFLD include the presence of bright hepatic echotexture (compared with the kidneys), deep attenuation, and vascular blurring either singly or in combination. ${ }^{23}$ Much work has been done on NAFLD and NASH in the western world but its presentation and aetiology has not been well studied in Asian population because of its asymptomatic presentation. This study was conducted to know the frequency of NAFLD in type 2 diabetic patients in Paki- 
stan and to see the differences in the Body mass index and biochemical markers in fatty liver disease patients and non fatty liver disease patients.

\section{MATERIAL AND METHODS}

It was an observational cross sectional study conducted at the medical outpatient department of Jinnah postgraduate medical centre, Karachi from September 2006 to March 2007. 120 diagnosed type 2 diabetic patients of both sexes were included in the study. The patients with known chronic liver disease (hepatitis B surface antigen or Anti HCV positive), pregnancy, and history of alcohol or drugs which cause fatty liver were excluded. Informed consent was taken from the subjects and they were evaluated by abdominal ultrasonography. Fatty liver was diagnosed on the basis of diffuse hyperechoic echo texture, increased echo texture compared with kidneys, vascular blurring and deep attenuation. The patients were divided into fatty liver and non fatty liver group and were further evaluated by the measurement of BMI, haemoglobin (HbA1c), total bilirubin, alanine aminotransferase (ALT), aspartate aminotransferase(AST) , alkaline phosphatase(AKP), gamma glutamyl transpeptidase (GGT), total cholesterol (TC) triglycerides (TG), low density lipoprotein (LDL) and high density lipoprotein (HDL). The data was entered and analyze into Statistical packages for social science (SPSS version 10.0). Frequency and percentages were computed for categorical variable were presented in pie graph. Mean and standard deviation were computed for quantitative variables like age, BMI, HbA1c, total bilirubin, AST, ALT, AKP, GGT, TC, TG, LDL and HDL. Independent sample $t$ test was used to compare mean of all the quantitative variables between the two groups of patients were 0.05 level was considered significant. Multivariate analysis was performed by using multiple logistic regression models for independent risk factors of fatty liver. $\mathrm{P}$ value less than 0.05 was considered statistically significant.

\section{RESULTS}

The study included 120 patients of type 2 diabetes mellitus. The average age of the patients was $50 \pm 7.8$ years (Ranging form 35 to 66). Forty-one (34.2\%) were males and $79(65.8 \%)$ were females; female to male ratio was 1.9:1. Seventy three (60.8\%) patients had fatty liver whereas forty seven (39.2\%) had no fatty liver on ultrasonography. The average liver size was $17.2 \pm 3.1 \mathrm{~cm}$ in fatty liver patients and $13 \pm 2.4$ $\mathrm{cm}$ in non fatty liver patients. Comparison of means of serum biochemical markers between fatty liver and non fatty liver groups is presented in Table I. The values of all these biochemical markers except HDL were elevated in fatty liver disease patients as non fatty liver disease group and the differences were found to be statistically significant. ( $P$ value $<0.01$ ). Multivariate logistic regression was used to select the variables independently associated with diabetic fatty liver. Defining as FLD as 1 and non FLD as 0, and the probability of fatty liver disease was presented as P-Values as shown in table-2. All markers entered the model of the logistic regression as independent risk factors of the diabetic fatty liver. Body mass index and triglycerides were positively correlated with fatty liver. (BMI: Odd ratio $=1.55 \mathrm{p}=0.03$; triglycerides: $\mathrm{OR}=1.04$ $p=0.035)$.

\section{FIGURE I:}

\section{FREQUENCY OF DIABETIC FATTY LIVER PATIENTS $(n=120)$}

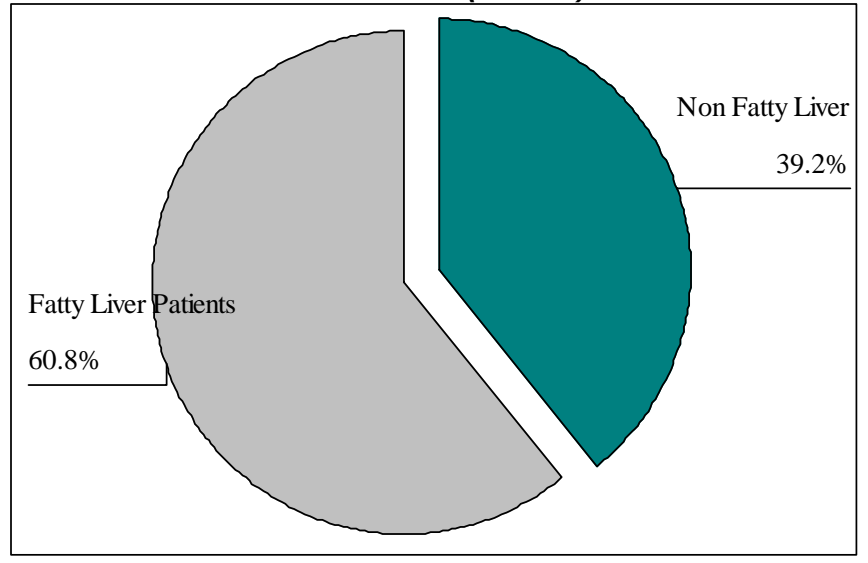

Fatty Liver Patients $\quad=73$

Non Fatty Liver Patients $=47$

\section{DISCUSSION}

Non alcoholic fatty liver disease is a common hepatic disorder characterized by accumulation of fat in the liver parenchyma of the patients who do not drink excessive amount of alcohol. It is a clinicopathological syndrome characterized by wide spectrum of liver damage ranging from benign fatty infiltration to steatohepatitis, advanced fibrosis and cirrhosis. ${ }^{2}$ The prevalence of NAFLD is high in conditions associated with insulin resistance, such as obesity, type-II diabetes mellitus, dyslipidemia and the metabolic syndrome. ${ }^{10}$ It is very common in type-II diabetic patients, with $50 \%$ to $75 \%$ subjects demonstrating fat in the liver by ultrasound. ${ }^{24}$ In our study the prevalence of fatty liver in type 2 diabetics was $60.8 \%$ which correlates with the prevalence reported in study conducted by Akbar and Kawther in Saudi Arabia i.e. 55\% (25) and by Gupta et al in India which was $49 \% .{ }^{15}$ The presence of NAFLD correlates significantly with both BMI and waist hip ratio. ${ }^{26,27}$ Though in our study only BMI was taken as a marker for obesity, raised BMI showed strong correlation with presence of fatty liver. The multiple logistic 
TABLE I:

COMPARISON OF LABORATORY ABNORMALITIES BETWEEN FATTY LIVER AND NON FATTY LIVER $(n=120)$

\begin{tabular}{|l|c|c|c|c|}
\hline \multicolumn{1}{|c|}{ Study Variables } & NAFL $\mathbf{n = 7 3}$ & No NAFL $\mathbf{n = 4 7}$ & t-value & P-Values \\
\hline BMI & $30.17 \pm 3.92$ & $23.7 \pm 2.55$ & 9.94 & $\mathrm{P}<0.01^{*}$ \\
\hline Hb A1c & $8.29 \pm 088$ & $7.02 \pm 0.47$ & 9.08 & $\mathrm{P}<0.01^{*}$ \\
\hline Bilirubin (mg/dl) & $0.97 \pm 0.24$ & $0.67 \pm 0.42$ & 3.84 & $\mathrm{P}<0.01^{*}$ \\
\hline AST $(\mathrm{mu} / \mathrm{ml})$ & $37.23 \pm 17.9$ & $20.38 \pm 5.7$ & 6.22 & $\mathrm{P}<0.01^{*}$ \\
\hline ALT $(\mathrm{mu} / \mathrm{ml})$ & $51.92 \pm 27.3$ & $26.47 \pm 8.59$ & 6.18 & $\mathrm{P}<0.01^{*}$ \\
\hline AKP $(\mathrm{mu} / \mathrm{ml})$ & $293.64 \pm 109.5$ & $162.21 \pm 39$ & 7.90 & $\mathrm{P}<0.01^{*}$ \\
\hline GGT & $43.37 \pm 19.92$ & $22.88 \pm 7.39$ & 6.75 & $\mathrm{P}<0.01^{*}$ \\
\hline Total Cholesterol & $198.12 \pm 47.6$ & $158.53 \pm 41.84$ & 4.66 & $\mathrm{P}<0.01^{*}$ \\
\hline TG $(\mathrm{mg} / \mathrm{dl})$ & $244.8 \pm 128.9$ & $117.49 \pm 39.29$ & 6.57 & $\mathrm{P}<0.01^{*}$ \\
\hline HDL $(\mathrm{mg} / \mathrm{dl})$ & $37.32 \pm 7.12$ & $43.98 \pm 6.94$ & -5.04 & $\mathrm{P}<0.01^{*}$ \\
\hline LDL $(\mathrm{mg} / \mathrm{dl})$ & $120.1 \pm 48.6$ & $90.72 \pm 34.63$ & 3.59 & $\mathrm{P}<0.01^{*}$ \\
\hline
\end{tabular}

*Significant

TABLE II:

MULTIPLE LOGISTIC REGRESSION ANALYSIS OF INDEPENDENT RISK FACTORS OF FATTY LIVER $(n=120)$

\begin{tabular}{|l|c|c|c|c|c|}
\hline Study Variables & Estimated & Wald & P-Value & OR & 95\%Cl \\
\hline Constant & -35.66 & 6.56 & 0.01 & - & - \\
\hline BMI & 0.436 & 3.04 & $0.03^{*}$ & 1.546 & $1.15-2.5$ \\
\hline Hb A1c & 1.934 & 1.32 & 0.25 & 6.914 & $0.26-185.6$ \\
\hline Bilirubin (mg/dl) & -4.097 & 2.12 & 0.145 & 0.017 & $0.0-4.1$ \\
\hline AST $(\mathrm{mu} / \mathrm{ml})$ & 0.064 & 0.312 & 0.577 & 1.066 & $0.85-1.3$ \\
\hline ALT $(\mathrm{mu} / \mathrm{ml})$ & -0.011 & 0.013 & 0.909 & 0.99 & $0.82-1.18$ \\
\hline AKP $(\mathrm{mu} / \mathrm{ml})$ & 0.028 & 3.17 & 0.075 & 1.028 & $0.99-1.06$ \\
\hline GGT & 0.076 & 1.06 & 0.304 & 1.078 & $0.93-1.24$ \\
\hline Total Cholesterol & -0.041 & 2.20 & 0.138 & 0.96 & $0.91-1.01$ \\
\hline TG $(\mathrm{mg} / \mathrm{dl})$ & 0.039 & 4.46 & $0.035^{\star}$ & 1.040 & $1.003-1.07$ \\
\hline HDL $(\mathrm{mg} / \mathrm{dl})$ & -0.009 & 0.006 & 0.937 & .991 & $0.79-1.24$ \\
\hline LDL $(\mathrm{mg} / \mathrm{dl})$ & 0.054 & 2.65 & 0.103 & 1.06 & $0.98-1.13$ \\
\hline
\end{tabular}

*Significant

KEY OR = Odd Ratio, $\mathrm{Cl}=$ confidence interval 
regression analysis showed $\mathrm{BMI}$ as the independent predictor of fatty liver ( $P$ value 0.03 ) In diabetic fatty liver group, the mean BMI was $30.17 \pm 3.92$ where as in non-fatty liver group it was $23.7 \pm 2.55$ ( $P$ value $<$ 0.01). In literature, among severely obese patients with diabetes, the prevalence of NAFLD has been found to be $100 \%{ }^{2,28}$ Although overall obesity is clearly associated with NAFLD, body fat distribution appears to play an important role in the pathogenesis of NAFLD, via both its strong association with insulin resistance and possibly as source of free fatty acids. Increased intra abdominal fat deposition is also associated with what are commonly considered to be clinical manifestation of insulin resistance including type-II diabetes mellitus, impaired glucose tolerance, dyslipidemia and the metabolic syndrome. ${ }^{29}$ The relationship between fatty liver, impaired glucose tolerance, diabetes mellitus and hyperlipidemia is well established. ${ }^{28,30}$ It has been demonstrated that insulin resistance leads to higher free fatty acid load to the liver, consequently higher triglyceride synthesis and increased secretion of triglyceride rich very low density lipoprotein (VLDL) from the liver. In fact, hypertriglyceridemia have been strongly correlated with liver fat accumulation. ${ }^{28,31}$ Our study showed increased triglyceride levels (mean $244.8 \pm 128.9)$ in diabetic fatty liver group as compared to non fatty liver group (triglycerides mean $43.98+6.94)$ and the results were statistically significant $(\mathrm{P}$ value $<0.01)$. In a logistic multiple logistic regression analysis high triglycerides were also found to be independent predictor of fatty liver in diabetic population ( $P$ value 0.35 ). These findings are consistent with those reported in the literature. ${ }^{32} \mathrm{Jin} \mathrm{HB}$ et al in a study conducted in China also found that fatty liver positively correlated with plasma triglyceride levels and negatively with plasma HDL-C level, but not with total cholesterol[33]. In our study also, the elevated total cholesterol did not positively correlated with fatty liver disease ( $P$ value 0.138). In addition, we also found that HbA1c levels in fatty liver disease group were higher then non-fatty liver group, which confirmed the obvious dysglycemia in these patients $(P$ value 0.01). Although patients with type-II diabetes mellitus have normal or increased levels of insulin, they are resistant to peripheral action of this hormone. ${ }^{34}$ This insulin deficiency, by promoting lipolysis, is an important cause of fatty liver in patients with poorly controlled diabetes mellitus. In this situation, massive lipolysis results in marked mobilization of free fatty acids, which are taken up by the liver and used for triglyceride synthesis with the consequent hepatic accumulation leading to steatosis. Free fatty acids are potentially cytotoxic causing mitochondrial swelling, increased lysosomal fragility, depression of enzyme activity, and impairment of membrane integrity. This cellular damage with subsequent inflammation may result in cellular death and fibrosis. Free fatty acids have also been found to be elevated in liver tissue of obese patients. Thus although diabetes mellitus and obesity may lead to increased liver fibrosis through different mechanism, the effect of these two conditions may be additive when they both exist in the same individual. ${ }^{28}$ The levels of ALT, AST, ALK, and GGT were also elevated in diabetic fatty liver patients in our study. This is also reported in other studies as well. ${ }^{35}$, ${ }^{36}$ In this study, we also noted the marked difference between the mean values of alkaline phosphatase in the two groups (293.64 Vs 162.21)( $\mathrm{P}$ value < 0.01).Though raised ALT levels are taken as the first marker of fatty infiltration of the liver $(29,36)$, raised ALT levels did not positively correlated with the presence of fatty liver in our study( $P$ value 0.909). Mofrad et al in their study reported that the histologic spectrum of NAFL is not significantly different in those with normal ALT from those with elevated ALT values and normal ALT levels does not guarantee freedom from steatohepatitis. ${ }^{37}$ The mechanism behind the elevated liver enzymes in NAFL patients is that dyslipidemia and insulin resistance leading to lipid deposition in hepatocytes causes induction of mitochondrial swelling, increased lysosomal fragility and impaired membrane integrity, resulting ultimately in the release of hepatic enzymes from the injured hepatocytes. ${ }^{28}$ Sorbi $D$ et al[38] showed that in patients with non-alcoholic fatty liver disease the ratio of AST to ALT is usually $<1$ and it has got potential value in differentiating NAFL from alcoholic liver disease. Our study also showed that in the majority of fatty liver patients AST to ALT ratio was $<1$. Our study has got few limitations. The diagnosis of NAFLD in our study was based on ultrasonography and exclusion of the known causes of chronic liver disease, but was not confirmed by liver biopsy. However, ultrasonography is by for the commonest way of diagnosing NAFL in clinical practice and can accurately identify steatosis with a sensitivity of $80 \%$ and specificity of $99 \%(22,23)$. Indeed, it has been reported that the presence of $>33 \%$ fat or liver biopsy is optimal for ultrasound detection of steatosis, whereas the sensitivity of ultrasonography is poor when hepatic infiltration is less than $33 \%{ }^{22}$ Our diagnosis of NAFLD, based on standard ultraonography may therefore overlook subjects with small amount of fat infiltration of the liver. In conclusion, the prevalence of non alcoholic fatty liver is high in type 2 diabetic patients and obesity, dyslipidemia, dysglycemia and elevation of liver enzymes is seen more frequently in fatty liver than in non fatty liver type 2 diabetic patients. The independently associated risk factors for diabetic fatty liver are the raised BMI and elevated levels of triglycerides. 


\section{REFERENCES}

1. Podolsky DK. Infiltrative, genetic and metabolic disease affecting the liver. In: Braunwald $E$, Hauser SL, Fauci AS, Longo DL, Kasper DL, Jameson JL; editors. Harrison's principles of internal medicine. $16^{\text {th }}$ ed. New York Mc-Graw Hill 2005: 1869 - 73 .

2. Angulo P. Non alcoholic fatty liver disease. N Eng J Med 2002; 346: 1221 - 31.

3. Mulhall BP, Ong JP, Younossi ZM. Non-alcoholic fatty liver disease. An overview. J Gasteroenterol Hepatol 2002; 11: 1136 - 43.

4. Harrison SA, Torgerson S, Hayashi PH. The natural history of non-alcoholic fatty liver disease a clinicohistopathological study. Am J Gastroenterol 2003;98:2042- 47.

5. Ekestedt M, Franzen LE, Mathiesen U, Thorelius L, Holmquist M, Bodemar $G$ et al. Long term follow up of patients with NAFLD and elevated liver enzyme. Hepatology 2006; 44: 865 - 75.

6. Maheshwari A, Thuluvath PJ. Cryptogenic cirrhosis and NAFLD: are they related. Am J Gastroenterol 2006; 101: $664-68$.

7. Davila JA, Morgan RO, Shaib Y, McGlynn KA, ElSerag HB. Diabetes increases the risk of hepatocellular carcinoma in the United States: a population basal case control study. Gut 2005; 54: $533-39$.

8. Hanley A, William K, Fiesta A, Wegenknecht L, D'Agostino R, Haffner S. Liver markers and development of the metabolic syndrome the insulin resistance atherosclerotic theory. Diabetes 2005; 54: $3140-47$.

9. Oh SY, Cho YK, Kong MS, Yoo TW, Park JH, Kim $\mathrm{HJ}$ et al. The association between increased alanine aminotransferase activity and metabolic factors in nonalcoholic fatty liver disease. Metabolism 2006; 55: $1604-9$.

10. Neuschawander-Tetri BA. Non-alcoholic steatohepatitis and the metabolic syndrome. Am J Med Sci 2005; 330: $326-35$.

11. Angelico F, Del DA, Conti R, Franciosio S, Feole $\mathrm{K}$, Fiorello $\mathrm{S}$ et al. Insulin resistance, the metabolic syndrome and non-alcoholic fatty liver disease. J Clin Endocrinol Metabol 2005; 90: 1578 $-82$.

12. Kelly DE, McKolanis TM, Hegasi RA, Kuller LH, Kalhan SC. Fatty liver in type-2 diabetes mellitus, relation to regional adiposity, fatty acids and insulin resistance. Am J Physiol Endcrinol Metab 2003; 285: 906 - 16.

13. Singh SP. Non alcoholic fatty liver disease. The unfolding monster? editorial. J Gastroenterol Hepatol 2006; 21: 199 - 201.
14. Choudhary J, Sanyal A. Clinical aspects of fatty liver disease. Semin Liver Dis 2004; 21: 349 - 62.

15. Gupte P, Amarapurkar D, Agal S, Baijal R, Kulshreshtta P, Pramik S et al. Non-alcoholic steatohepatitis in type 2 diabetes mellitus. J Gastroenterol Hepatol 2004; 19: $854-8$.

16. Shen L, Fan JG, Shao Y, Zeng MD, Wang JR, Luo $\mathrm{GH}$ et al. Prevalence of non-alcoholic fatty liver among administrative officers in Shinghai: an epidemiological survey. World J Gastroenterol 2003; 9: 1106 - 10.

17. Qari FA, Al Ghamdi A. Fatty liver in overweight and obese patients in Western part of Saudi Arabia: a study of sonological prevalence. Pak J Med Sci 2005; 21: $143-7$.

18. Chen Q, Chen H, Huang K, Zhong Y, Han J, Zhu $Z$ et al. Clinical features and risk factor of patients with fatty liver in Guanzhou area. World J Gasteroenterol 2004; 10: 899 - 92.

19. Khuram M, Abdul S, Arshad MM, Khaar H, Hasan Z. Characteristic features of NAFLD patients. Rawal Med J 2004; 29: 8 - 12.

20. Madan K, Batra Y, Gupta SP, Chander B, Rajan KD, Tewatia MS et al. Nonalcoholic fatty liver disease may not be a severe disease at presentation among Asian Indians. World J Gasteroenterol 2006; 12: $3400-5$.

21. Falck-Ytter Y, Younossi ZM, Marchesini G, McCullough AJ. Clinical features, and natural history of non-alcoholic steatosis syndromes. Semin Liver Dis 2001; 21: $17-26$.

22. Saadeh S, Younossi ZM, Remer EM, Gramlich T, Ong JP, Hurley $M$ et al. The utility of radiological imaging in nonalcoholic fatty liver disease. Gastroenterology 2004; 99: 1316-20.

23. Siegelman ES, Rosen MA. Imaging of hepatic steatosis. Semin. Liv. Dis. 2001: 21: 71-80

24. Bacon BR, Farahvash MJ, Janney CG. Neuschwander Tetri BA. Non-alcoholic steatohepatitis:an expanded clinical entity.Gasteroenterology1994;107:1103 - 9.

25. Akber $\mathrm{DH}$, Kawther AH. Non-alcoholic fatty liver disease in Saudi type-II diabetic subjects attending a medical outpatient clinic. Diabetes Care 2003 ;26: 3351 - 65.

26. Rocha R, Cotrim HP, Carvello FM, Sequira AC, Brega $H$, Freitas LA. Body mass index and waist circumference in nonalcoholic fatty liver disease. $\mathrm{J}$ Hum Nutr Dietet 2005; 18: 365 - 70.

27. Salgodo Jr. W, Santos JS, Sankarankutty AK, Castro ESilva OD. Non-alcoholic fatty liver disease and obesity. Acta Cir Bras 2006; 21: 72 78.

28. Angulo P, Keach JC, Batts KP, Lindor KD. Independent predictor of liver fibrosis in patients with 
nonalcoholic steatohepatitis. Hepatology 1999; 30: $1356-62$.

29. Miyazaki Y, Glass L, Triplett C, Wajcberg E, Mandarino LJ, De Fronzo RA Abdominal fat distribution and peripheral and hepatic insulin resistance in type-II diabetes mellitus. Am J Physiol Endocrinol 2002; 283: 1135 - 43.

30. Luyckx FH, Lefebve PJ, Scheen AJ. Non-alcoholic steatohepatitis: association with obesity and insulin resistance, and influence of weight loss. Diabetes Metab 2000; 26: 98 - 106.

31. Donnelly KL, Smith Cl, Schwarzenberg SJ, Jessurun J, Boldt MD, Parks EJ. Sources of fatty acids stored in liver and secreted via lipoproteins in patients with non alcoholic fatty liver disease. J Clin Invest 2005; 115: 1343-51.

32. Sharabi $Y$, Eldad A . Non alcoholic Fatty liver disease is associated with hyperlipidemia and obesity. Am J Med 2000;109: 171

33. Jin HB, Gu ZY, Yu CH, Li YM. Association of nonalcoholic fatty liver disease with type-II diabetes: clinical features and independent risk factors in diabetic fatty liver patients. Hepatobiliary Pancreat Dis Int 2005; 4: 389 - 92.
34. Raz I, Eldor R, Cernea S, Shafrir E. Diabetes: insulin resistance and derangements in lipid metabolism. Care though intervention in fat transport and storage. Diabetes Metab Res Rev 2005; $21: 3$ $-14$.

35. Deng HM, Xiao CQ, Par HL. Analysis of associated factors in type-II diabetic patients with fatty liver. J Clin Intern Med 2003; 20: 22 - 25.

36. Vazarova B, Stefan N, Lindsay RS, Saremi A, Pratley RE, Bogardus $C$ et al. High alanine aminotransferase is associated with decreased hepatic insulin sensitivity and predicts the development of type-II diabetes. Diabetes 2002; 51: 1889 $-95$.

37. Mofrad $P$, Contos $M J$, Haque $M$, Sargeant $C$, Fisher RA, Luketic VA. Clinical and histologic spectrum of nonalcoholic fatty liver disease associated with normal ALT values. Hepatology 2003;37:1286-92.

38. Sorbi D, Boynton J, Lindor KD. The rates of aspartate aminotranferase to alanine aminotranferase: potential valve in differentiating nonalcoholic steatohepatitis from alcoholic liver disease. Am J Gastroenterol 1999; 94: 1018 - 22.

\begin{tabular}{|l|}
\multicolumn{1}{c|}{ AUTHOR AFFILIATION: } \\
Dr. Shobha Luxmi (Corresponding Author) \\
Tranie FCPS-II \\
Jinnah Postgraduate Medical Centre \\
Karachi, Sindh-Pakistan. \\
Email: sluxmi77@yahoo.com \\
Dr. Rukhsana Abdul Sattar \\
Associate Professor \\
Jinnah Postgraduate Medical Centre \\
Karachi, Sindh-Pakistan. \\
Prof. Jamal Ara \\
Department of Medicine \\
Jinnah Postgraduate Medical Centre \\
Karachi, Sindh-Pakistan. \\
\hline
\end{tabular}

